\title{
Cytapheresis in Inflammatory Bowel Diseases: Current Evidence and Perspectives
}

\author{
Silvio Danese ${ }^{\mathrm{a}}$ Erika Angelucci ${ }^{\mathrm{b}}$ Tommaso Stefanelli ${ }^{\mathrm{a}}$ Paolo Omodei $^{\mathrm{a}}$ \\ Carmelo Luigiano $^{a}$ Silvia Finazzia Nico Pagano ${ }^{a} \quad$ Alessandro Repici $^{a}$ \\ Maurizio Vecchic Alberto Malesci ${ }^{\mathrm{a}}$ \\ ${ }^{a}$ Division of Gastroenterology, Istituto Clinico Humanitas, IRCCS in Gastroenterology, University of Milan, \\ Rozzano/Milan, ${ }^{\mathrm{b}}$ Division of Gastroenterology, Policlinico Umberto I, Università Sapienza, Rome, and \\ 'Division of Gastroenterology, IRCCS-Policlinico San Donato Milanese, University of Milan, Milan, Italy
}

\begin{abstract}
Ulcerative colitis and Crohn's disease are inflammatory bowel diseases with a chronic relapsing course. Management of both conditions is far from being fully satisfactory. For this reason in the last decade a large number of biological therapies, targeting cytokines involved in intestinal inflammation, has been developed with various results in terms of efficacy, safety and costs. Activated granulocytes and monocytes represent the major sources of pro-inflammatory cytokines in the intestinal mucosa, playing a pivotal role in inducing and maintaining intestinal inflammation. Leukocytapheresis using an adsorptive carrier-based system (Adacolumn ${ }^{\circledR}$ ) or a removal filter column (Cellsorba ${ }^{\circledR}$ ) has been proposed as a feasible, safe and effective therapy for ulcerative colitis and Crohn's disease. The objective of this paper is to provide an overview on the current knowledge about mechanisms of action, available clinical data and the possible future perspectives for the use of Adacolumn and Cellsorba in the management of inflammatory bowel diseases.
\end{abstract}

Copyright $\odot 2008$ S. Karger AG, Basel

\section{Introduction}

Inflammatory bowel diseases (IBD) are chronic inflammatory disorders of the gastrointestinal tract with a chronic relapsing course. While mild forms of IBD are usually successfully treated with aminosalicylates for maintenance of remission and corticosteroids for flaresup, management of moderate and severe forms of IBD is far from being fully satisfactory, especially for the many cases of steroid dependency, steroid resistancy and steroid intolerance [1].

A large number of different therapeutic approaches have been tried in the last decade, mainly with biological therapies targeting cytokines involved in intestinal inflammation. They have been approved for the treatment of moderate and severe forms of both Crohn's disease (CD) and ulcerative colitis (UC), with various results in terms of efficacy, safety and costs.

Although the etiology of IBD is unknown, a crucial pathogenic role is attributed to leukocytes. Activated granulocytes and monocytes represent the major source of pro-inflammatory cytokines in the intestinal mucosa and play a pivotal role in inducing and maintaining intestinal inflammation $[2,3]$. Lately, the apheresis (which means 'to take away') of leukocytes from peripheral blood using extracorporeal circulation has been proposed as a feasible, safe and effective therapy for IBD.

Three types of leukocytapheresis using extracorporeal circulation have been developed.

Centrifugal leukocytapheresis, also called lymphocytes removal apheresis, was first used in IBD patients with active CD in 1980s [4]. This device was able to re-

\section{KARGER}

Fax +4161306 1234 E-Mail karger@karger.ch www.karger.com
(C) 2008 S. Karger AG, Basel

0012-2823/08/0772-0096\$24.50/0

Accessible online at:

www.karger.com/dig
Silvio Danese, MD, Division of Gastroenterology

Istituto Clinico Humanitas - IRCCS in Gastroenterology

Viale Manzoni, Rozzano, IT-20089 Milan (Italy)

Tel. +39028224 4771, Fax +390282245101

E-Mail sdanese@hotmail.com 
move from peripheral whole blood about $55 \%$ of lymphocytes but also $40 \%$ of granulocytes and many red blood cells and platelets.

More recently the use of a fiber filter or column to selectively remove specific components of peripheral whole blood has replaced the simple centrifugation apheresis. To date, two methods are available for selective leukocytapheresis in patients with IBD or rheumatological conditions. In the first one, Adacolumn ${ }^{\circledR}$ (Japan Immunoresearch Laboratories Co., Ltd) provides an apheresis column filled with cellulose acetate beads which act as selective adsorptive carriers for granulocytes and monocytes, but not for lymphocytes and platelets [5]. In the second one, Cellsorba ${ }^{\circledR}$ (Asahi Medical, Tokyo, Japan), the adsorptive column is made of non-woven polyester fiber filter and is able to remove about $90-100 \%$ of granulocytes and monocytes, $30-60 \%$ of lymphocytes and a certain amount of platelets $(30 \%$ in the first $30 \mathrm{~min}$ of the session) from peripheral blood $[6,7]$.

Continuous injection of anticoagulant, nafamostat mesilate or heparin, is carried out to prevent coagulation of blood during extracorporeal circulation.

In a recent comparative study evaluating the use of these two different anticoagulants during leukocytapheresis, the use of heparin was associated to a significantly lower frequency of adverse events as compared to nafamostat mesilate [8].

The objective of this paper is to provide an overview on the mechanisms of action, the available clinical data and the possible future perspectives for the use of Adacolumn and Cellsorba in the management of IBD.

\section{The Adacolumn Leukocytapheresis System: Granulocytes-Monocytes Apheresis}

The Adacolumn is a medical device for cell removal (cytapheresis) in which activated granulocytes and monocytes/macrophages are selectively depleted from venous blood using an extracorporeal circulatory system with a G-1 column (Japan Immunoresearch Laboratories Co., Ltd). This column has a capacity of $335 \mathrm{ml}$ and is filled with $220 \mathrm{~g}$ cellulose acetate beads (about 35,000 pieces) of $2 \mathrm{~mm}$ in diameter and bathed in sterile saline. These carriers selectively adsorb granulocytes, monocytes/macrophages together with a small amount of lymphocytes. Granulocytes-monocytes apheresis (GMA) with Adacolumn needs two venous accesses with 18-gauge needles, e.g. at the antecubital (or femoral) vein on one hand for blood supply into the G1 column and on the other arm for returning the blood back to the patient. The blood pumped out from patients' veins is anticoagulated by heparin. The anticoagulated blood is passed through the G1 column at a flow rate of $30 \mathrm{ml} / \mathrm{min}$. The duration of each session is about $60 \mathrm{~min}$. GMA is mainly performed once weekly for 5 consecutive weeks, but different protocols with 2 sessions/week or other schedules have been tested. Adhesion and absorption of peripheral blood cells begin when the blood comes into contact with the cellulose acetate beads in the column. The cellulose acetate beads bind to immune complexes (IC) and immunoglobulin G (IgG) from the plasma and trigger the complement cascade, generating active complement fragments such as $\mathrm{C} 3 \mathrm{a}, \mathrm{C} 3 \mathrm{~b}$ and C5a $[5,9,10]$. After adsorption to the beads, binding sites on IgG and IC are available for Fc $\gamma$ receptors (FcyRs) on granulocytes and monocytes. The adsorbed opsonins also serve as binding sites for leukocyte complement receptors. The carriers adsorb about $65 \%$ of the incoming granulocytes, $55 \%$ of monocytes/macrophages and only $2 \%$ of lymphocytes. All adsorbed cells are complement receptor- and Fc $\gamma \mathrm{R}$-bearing leukocytes. The systemic number of lymphocytes, erythrocytes and platelets did not show any significant changes after GMA [5, 9]. Depletion of activated granulocytes and monocytes/macrophages reduces leukocyte-dependent inflammations in immunomediated disorders, such as rheumatologic conditions and IBD. Except for small subpopulations, lymphocytes do not express Fc $\gamma \mathrm{R}$ and complement receptors. This explains the scarce adsorption of lymphocytes to G1 column carriers.

However, the selective removal of granulocytes and monocytes/macrophages from peripheral blood is most probably not the exclusive mechanism responsible for the clinical efficacy of GMA. The following results, observed in vitro and in vivo, are related to the mode of action of GMA, although it is not yet fully understood:

- An increased number of CD10-negative immature neutrophils from the bone marrow pool is seen shortly after apheresis start. Immature neutrophils have less pro-inflammatory properties than mature neutrophils [11].

- A decreased release of pro-inflammatory cytokines such as tumour necrosis factor $\alpha(\mathrm{TNF} \alpha)$, interleukin (IL)- 6 , IL- 8 and IL-1 $\beta$ by peripheral blood leukocytes has been observed after Adacolumn sessions [12-14].

- Lower circulating levels of L-selectin and increased levels of CD11b/CD18 (Mac-1) have been reported after GMA sessions [15], followed by reduced leukocyte rolling on the vascular endothelium and reduced migration of granulocytes to sites of inflammation $[16,17]$. 
Fig. 1. Adacolumn ${ }^{\circledR}$ mechanism of action: selective adsorption/removal of granulocytes and monocytes to the cellulose acetate beads.

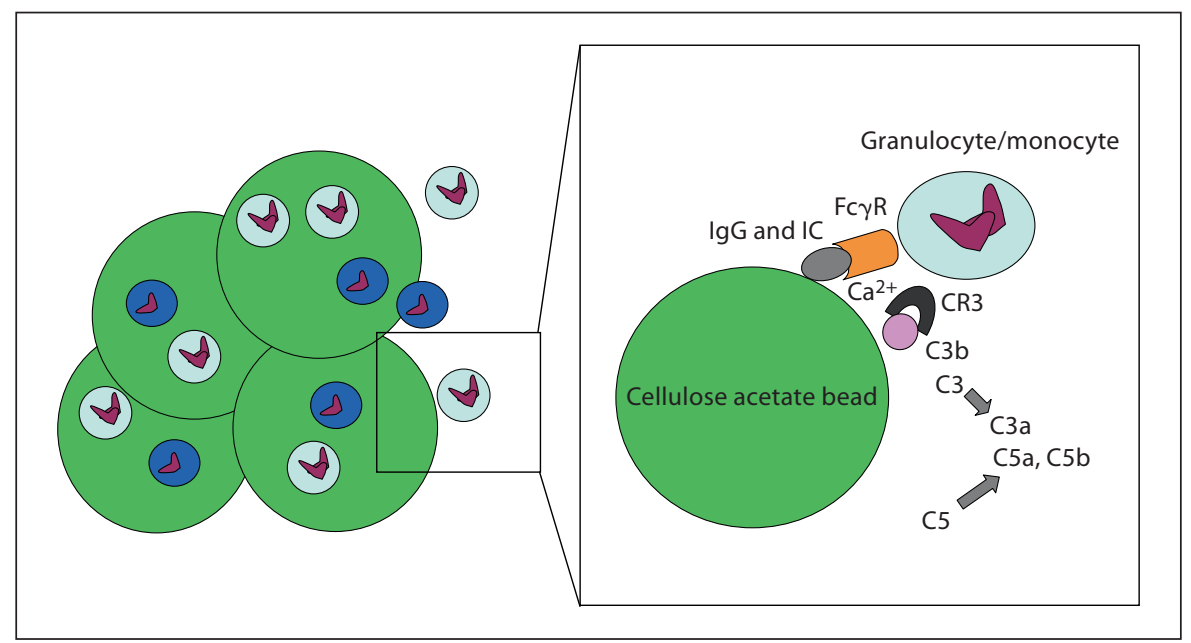

- Upregulation of Mac-1 after Adacolumn treatment has also been found. It is known to promote apoptosis of granulocytes [18].

- An increased production of anti-inflammatory IL-1 receptor antagonist (IL1-ra) $[19,20]$ and hepatocyte growth factor [21] were seen.

- High levels of shedded, soluble TNF $\alpha$ receptors type I (p55, RI) and type II (p75, RII) are released during GMA [22].

- Expression of Toll-like receptor 2 (TLR2) on granulocytes also appears to be down-regulated after GMA, both in vivo and in vitro [23].

- An increase in number of peripheral CD25highCD4+ regulatory $\mathrm{T}$ cells [24] and a decrease in number of highly pro-inflammatory $\mathrm{CD} 14+\mathrm{CD} 16+$ monocytes [25] were seen.

These effects may jointly or independently contribute to the observed clinical efficacy of Adacolumn in the treatment of IBD. Mechanisms of action of Adacolumn are briefly summarized in figure 1.

\section{Adacolumn Leukocytapheresis for Ulcerative Colitis}

To date, a lot of case reports and open-label studies evaluated feasibility, safety and efficacy of Adacolumn apheresis for the treatment of UC patients. Results of trials evaluating the efficacy of Adacolumn in the treatment of UC are summarized in table 1 . No severe adverse events were reported in the following studies.

In a first open clinical trial involving 14 centers throughout Japan, 53 UC patients were selected for Adacolumn treatment $[12,26]$. Out of 105 eligible patients, 53 were in group I and received apheresis treatment, while 52 were in group II and continued conventional therapy. Out of the 53 patients in group I, 34 had moderate UC and 19 had severe UC; 11 of the 53 patients were at their first attack of UC. Each patient received 1 apheresis session for week per 5 consecutive weeks. At baseline, all patients were receiving prednisolone at a median dose of $24.4 \pm$ $3.6 \mathrm{mg} /$ day. At week 7, 31 of 53 (58.5\%) UC patients were considered responders to apheresis treatment (10/19 patients with severe attack and 21/34 patients with moderate attack); 11/31 responders achieved remission. At week 7 , the median dose of prednisolone was $14.2 \pm 2.25 \mathrm{mg}$. In group II, the median dose of prednisolone was increased to $63.1 \pm 13.82 \mathrm{mg} /$ day to achieve remission. 23 of 52 patients responded to treatment, and 7 of 23 responders achieved remission. As far as adverse events are concerned, 8 non-severe adverse events occurred in 5/53 patients in group I and 40 adverse events occurred in $24 / 52$ patients in group II, requiring discontinuation of treatment in 3 patients $[12,26]$.

A number of case reports or case series showed the efficacy and the good safety profile of Adacolumn in the treatment of UC patients unresponsive and/or dependent on conventional treatment [13, 27-30] (table 1).

In a prospective, open, uncontrolled study, 31 patients with severe steroid-refractory UC and 8 severe steroidnaive UC patients were assigned to Adacolumn treatment [31]. Each patient received a basic treatment course (5 apheresis sessions with 1 session/week) followed by further 5 sessions after a 1-week interval. Patients with a CAI $\geq 15$ points received 2 sessions of apheresis per week in the first 3 weeks, with a maximum of 11 sessions per patient. At week 12, 32/39 UC patients (82\%) achieved clin- 
Table 1. Studies evaluating efficacy of Adacolumn in the treatment of adult/pediatric UC patients ('overall response' includes both response and remission rate)

\begin{tabular}{lllcc}
\hline Group (first author) & Year & Indication & Patients & Overall response, \% \\
\hline Shimoyama [12, 26] & 2001 & Moderate-to-severe UC, refractory to conventional drugs & 53 & $58(31 / 53)$ \\
Hanai [31] & 2003 & Steroid-refractory UC & 31 & $87(27 / 31)$ \\
& & Steroid-naive UC & 20 & $100(8 / 8)$ \\
Suzuki [32] & 2004 & Steroid-naive UC & 44 & $85(17 / 20)$ \\
Naganuma [33] & 2004 & Steroid-refractory or -dependent UC & $46(33 / 44)$ \\
Hanai [34] & 2004 & Steroid-dependent UC & 30 & $94(43 / 46)$ \\
Yamamoto [35] & 2004 & Active distal UC & $14\left(13^{1}\right)$ & $87(26 / 30)$ \\
Domenech [36] & 2004 & Steroid-dependent UC & 60 & $76(10 / 13)$ \\
Kanke [37] & 2004 & Active steroid-naive/refractory/dependent/free UC & $83(50 / 60)$ \\
Kim [38] & 2005 & Moderate-to-severe UC, refractory to conventional drugs & 27 & $70(19 / 27)$ \\
Kruis [39] & 2005 & Steroid-dependent UC & $39\left(35^{1}\right)$ & $43(15 / 35)$ \\
D'Ovidio [40] & 2006 & Steroid-dependent/refractory UC & 12 & $100(12 / 12)$ \\
Bresci [41] & 2006 & Steroid-refractory UC & 8 & $100(8 / 8)$ \\
Sands [42] & 2006 & UC refractory to conventional drugs & 15 & $40(6 / 15)$ \\
Sakuraba [43], abstract & 2005 & Moderately active UC & 45 weekly & $47(21 / 45)$ \\
& & & 52 semi-weekly & $73(38 / 52)$ \\
Tanaka [44], abstract & 2006 & Steroid-dependent UC & 12 & $58(7 / 12)$ \\
& & Steroid-naive UC & 17 & $100(17 / 17)$ \\
Hanai [45], abstract & 2006 & Severe UC & 33 semi-weekly & 54 \\
Sakuraba [46], abstract & 2006 & Active pouchitis & 6 & $67(4 / 6)$ \\
Tomomasa [53] & 2003 & Pediatric UC steroid-refractory & 12 & $67(8 / 12)$ \\
Ikeda [54] & 2006 & Pediatric UC steroid-refractory & 4 & $75(3 / 4)$ \\
\hline
\end{tabular}

${ }^{1}$ Number of patients included in the final analysis.

ical remission, with $25 / 31 \quad(81 \%)$ steroid-refractory patients (median CAI $=3$ ) and $7 / 8(88 \%)$ steroid-naive patients (median CAI $=1$ ). Eleven non-severe adverse events occurred in 7 patients. No patient discontinued apheresis treatment due to adverse events. At 1 year, 26/32 responders maintained remission. 20 of these patients were on azathioprine/6-mercaptopurine, 7 received further apheresis sessions (1 session every 2 weeks), while the remaining patients continued on mesalamine.

Subsequently, a further open study was carried out to evaluate the safety and efficacy of Adacolumn in steroidnaive patients [32]. 20 steroid-naive patients with active UC were enrolled in the study. Only $6 / 20$ patients had a severe attack of UC, while the remaining 14 patients had a moderate attack. Each patient received a maximum of 10 sessions of apheresis ( 2 sessions/week) for up to 5 consecutive weeks. One week after the last session, 17/20 (85\%) patients were in clinical remission and mean CAI was 3. Half of the patients maintained the remission state at 8 months of follow-up with mesalamine treatment. Only 2 non-severe adverse events (self-limited headache) occurred in 2 patients during apheresis treatment.
An open study evaluated the safety and efficacy of Adacolumn in 44 steroid-refractory or -dependent active UC patients [33]. 14 of 44 patients had a severe attack of UC (CAI $\geq 12$ ), while $30 / 44$ patients had a moderate attack of the disease. 20 of 44 patients were defined as steroid-refractory, 10/44 patients were defined as steroid-dependent, and the remaining 14 patients were selected for Adacolumn because of their refusal to a further cycle of steroids at the time of relapse. Only 5/44 patients were on azathioprine/6-mercaptopurine at the study entry. Each patient received 1 apheresis session per week for 5 consecutive weeks, except for 6 patients with severe steroidrefractory UC who received 2 sessions/week in the first week of treatment and then 1 session/week. Overall, 33/44 (75\%) patients responded to Adacolumn treatment; $24 / 44$ (55\%) patients achieved clinical remission (CAI $\leq 3), 9 / 44$ (20\%) patients a clinical response (a decrease in CAI $\geq 3$ points but CAI remained $>4$ ), and 11/44 (25\%) patients had no response or aggravation. The remission rate ranged between $45 \%$ in the steroid-refractory group and $60 \%$ in the steroid-dependent group. The response rate (considering both remission and clinical response) varied 
from $60 \%$ in the steroid-refractory group to $90 \%$ in the steroid-dependent group [33].

In a further open study, 69 steroid-dependent UC patients were randomized to receive Adacolumn treatment (group I, $\mathrm{n}=46$ ) or an increased dosage of prednisolone (group II, $n=23$ ) [34]. 13 of $46(28.3 \%)$ patients in group I had severe disease $(\mathrm{CAI} \geq 12)$ compared to $4 / 23(17.4 \%)$ in group II. Each patient in group I received 1 apheresis session per week over 10 weeks, except for patients with severe UC who received 2 sessions/week in the first 3 weeks of treatment and then 1 session/week for a maximum of 11 sessions of apheresis. The mean baseline dose of prednisolone was $16.6 \pm 5.5 \mathrm{mg} /$ day in group I, and $29.8 \pm 13 \mathrm{mg} /$ day in group II $(\mathrm{p}<0.001)$. At weeks 6,12 and 24 , the remission rates in group I were 52,83 , and $85 \%$, respectively, compared to 61,65 , and $70 \%$ in group II at the same time. At week 24, the median dosage of prednisolone was $5.0 \pm 4.9 \mathrm{mg} /$ day in group I and 12.2 \pm 9.8 in group II $(\mathrm{p}=0.003)$, with a steroid-sparing effect due to Adacolumn treatment [34].

The clinical efficacy of Adacolumn has also been assessed in 30 patients with active distal UC [35]. Each patient received 1 apheresis session per week over 5 consecutive weeks. After 5 sessions, 21/30 (70\%) patients achieved clinical remission, with a significant decrease in DAI from 6 at baseline to 2 ( $p<0.0001$ ). Nine non-severe adverse events occurred in 8 patients during apheresis treatment; no patient discontinued treatment due to adverse events.

In a first Spanish open experience, 14 steroid-dependent active UC patients received $60 \mathrm{mg}$ /day of oral prednisolone, followed 1 week later by the adjunct of 1 Adacolumn session per week for 5 consecutive weeks [36]. At baseline, 9/14 UC patients were on azathioprine. At week $6,8 / 13(62 \%)$ patients achieved clinical remission and 2 additional patients had a clinical improvement. Of 8 patients, 6 maintained clinical remission over a mean follow-up period of 12.6 months. Only minor adverse events occurred during the study.

A different schedule of treatment with Adacolumn was evaluated in 60 active UC patients in a Japanese study [37]. 6 of 60 (10\%) patients were steroid-naive, 28/60 (46.7\%) were steroid-dependent, 14/60 (23.3\%) were steroid-refractory, and 12/60 (20\%) were steroid-free. Each patient received apheresis treatment according to different protocols: 1 session/week of $60 \mathrm{~min}(\mathrm{n}=9 ; 4 / 9$ patients were on prednisolone) or $90 \mathrm{~min}(\mathrm{n}=8 ; 4 / 8$ patients were on prednisolone) for 12 weeks; 2 sessions/week of $60 \mathrm{~min}$ ( $\mathrm{n}=19 ; 12 / 19$ patients were on prednisolone) or $90 \mathrm{~min}$ $(\mathrm{n}=24 ; 17 / 24$ patients were on prednisolone) for 5 weeks.
In the 42 patients who were on prednisolone at baseline, the dosage of steroids was tapered during the study. Two weeks after the last session, 50/60 (83.3\%) patients had clinical response. Among responders, 14 patients achieved clinical remission. Among the 28 steroid-dependent patients, 19 (67.9\%) became steroid-free. Improvement was maintained over a median period of 199 days (range 21-614), with time to relapse negatively correlated with endoscopic findings $(r=-0.488 ; p=0.0026)$ and cumulative dose of steroids $(\mathrm{r}=-0.325 ; \mathrm{p}=0.023)$. Apheresis twice a week seemed to be superior to 1 session/week for UCAI improvement $(p=0.023)$. Furthermore, twice per week or $90 \mathrm{~min}$ was superior to once per week or $60 \mathrm{~min}$ for improvement of symptoms $(\mathrm{p}=0.006)$. No serious adverse events were observed. 11 of 60 cases (18.3\%) showed mild headache and nausea, probably due to the use of nafamostat mesilate as anticoagulant instead of heparin [37].

In a Korean study, 27 patients with moderately (55.6\%) to severely (44.4\%) active UC, refractory to conventional treatment, received 5 apheresis sessions for 5 consecutive weeks [38]. At week 7, 70.4\% of patients showed overall improvement and in $56.3 \%$ of users steroids were tapered down or discontinued. No severe adverse events were observed.

In a second European experience, 39 steroid-dependent active (CAI 6-8) UC patients received 5 apheresis sessions over 5 consecutive weeks [39]. Patients with severe UC (CAI >8) were excluded from the study. 35 of 39 patients were included in intention-to-treat analysis. 13 of $35(37.1 \%)$ patients and 20/31 (65\%) patients achieved clinical remission $(\mathrm{CAI}<4)$ at 6 weeks and at 4 months, respectively. At week 6, 10/35 (28.6\%) patients also achieved endoscopic remission. A total of 46 adverse events occurred during the study, including 3 severe adverse events: thrombosis of the lower leg, significant drop in hemoglobin, and worsening of UC [39].

Two recent Italian open studies confirmed the safety and efficacy of Adacolumn in the treatment of steroiddependent and -refractory UC patients [40,41]. In one of these studies, 12 mildly to moderately active UC patients received 5 sessions of Adacolumn over 5 consecutive weeks [40]. 6 of $12(50 \%)$ patients had pancolitis. 9 of 12 patients were steroid-dependent and $3 / 12$ patients were steroid-refractory. At week 6, 8/12 patients achieved clinical remission $(\mathrm{CAI}<4)$, and $9 / 12$ patients discontinued steroid therapy. In the other Italian study, 8 steroid-refractory active UC patients with pancolitis received 5 sessions of apheresis over 5 consecutive weeks [41]. At week $6,8 / 8(100 \%)$ UC patients achieved both clinical and en- 
doscopic remission, and 6/8 maintained clinical remission at 12 months of follow-up. No serious adverse events were reported.

In the first US pilot feasibility study, a total of 15 patients with moderate $(n=10)$ to severe $(n=5)$ active UC received 5 sessions of Adacolumn over 5 consecutive weeks [42]. Of the 10 patients completing the study, at week 7, $5(50 \%)$ patients were responders and 1 patient achieved remission. A total of non-severe transient adverse events occurred during the study.

As far as the studies presented in recent editions of $D i$ gestive Disease Week and United European Gastroenterology Week are concerned, the efficacy and safety of weekly (1 session/week) GMA therapy have been compared to semi-weekly (2 sessions/week) GMA therapy for active UC [43]. Semi-weekly treatment with Adacolumn seemed to be more effective than weekly treatment and equally safe [43]. Two other studies published only as abstract evaluated the efficacy of Adacolumn in the treatment of moderate-severe and severe UC [44, 45]. Results from these studies have suggested that Adacolumn may represent a new 'rescue' therapy in patients with severe UC.

Lastly, a preliminary open trial evaluated the efficacy of Adacolumn in management of pouchitis [46]. 6 patients with active pouchitis received a total of 10 apheresis sessions. Of six patients, 4 improved after Adacolumn treatment.

\section{Adacolumn Leukocytapheresis for Crohn's Disease}

Adacolumn has also been proposed as an effective treatment for $\mathrm{CD}$. To date, only a few case series and open studies have evaluated the efficacy of Adacolumn in CD, with variable results (table 2). No severe adverse events were reported in these studies.

In a first report, 7 patients with active $C D$ refractory to conventional treatments received 1 Adacolumn session/week for 5-6 consecutive weeks [47]. 5 of 7 patients achieved clinical remission. Crohn's Disease Activity Index (CDAI) score fell from $244.7 \pm 50.4$ at baseline to $103.3 \pm 8.4(\mathrm{p}<0.01)$ after Adacolumn treatment. In the 5 patient responders, remission was maintained over 1 year with conventional medications (in 3 patients) or continuing Adacolumn treatment, 1 session/month (in 2 patients).

In a further open study, 6 patients with active $C D$ (CDAI 200-400 points) refractory to several conventional treatments, including enteral nutrition, received 1 Adacolumn session/week for 5 consecutive weeks (first se- ries of treatment) [48]. The patients responding to the first series (with a decrease of CDAI of at least 50 points from baseline) received a second series of Adacolumn treatment. Of 6 patients, 4 had lesions involving the colon and the ileum, whereas $2 / 6$ patients had lesions limited to the colon. All the patients enrolled had received enteral nutrition, only $2 / 6$ patients had received oral prednisolone before enrolment in the study. After the first series, 3/6 (50\%) patients responded to Adacolumn treatment, and 1 patient (16.7\%) achieved clinical remission. Mean CDAI fell from $258.2 \pm 36.3$ at baseline to $193.2 \pm 71.2$ at week $5(\mathrm{p}<0.03)$ and $166.5 \pm 16.6$ at week $7(\mathrm{p}<0.02)$. The only patient receiving the second series of treatment did not reach clinical remission after 14 weeks.

In a larger study, 21 patients with active CD (CDAI 200-399) refractory to conventional treatments, including enteral nutrition, received 1 Adacolumn session/week for 5 consecutive weeks [49]. At week 7, treatment was considered effective in 11/21 (52.4\%) patients. The CDAI score fell from $275.6 \pm 54.2$ at baseline to $214.8 \pm 89.2$ ( $\mathrm{p}=0.0005)$ after Adacolumn treatment.

In a Spanish study, 12 patients with active (CDAI $>150)$ steroid-dependent CD received 1 Adacolumn session/ week for 5 consecutive weeks [36]. 8 of 12 patients had non-stricturing non-penetrating $\mathrm{CD}$, whereas $4 / 12$ patients had penetrating $\mathrm{CD}$. Immunosuppressive drugs (azathioprine and methotrexate) and biological therapies (infliximab) had failed in 6 and 3 patients, respectively. 10 of 12 patients were included in the final analysis. At week 6, 7/10 (70\%) patients achieved clinical remission $(\mathrm{CDAI}<150)$, but only 1 patient maintained remission at 12 months of follow-up.

More recently in an Italian study, 6 patients with active (CDAI $213.3 \pm 32)$ steroid-resistant ileal CD received a 5 -session treatment with Adacolumn (1 session/week) [41]. At week 6, 5/6 patients achieved clinical remission and were steroid-free. The mean CDAI decreased from $213.3 \pm 32$ at baseline to $135 \pm 50(\mathrm{p}<0.015)$. At 12 months of follow-up only 2 patients maintained clinical remission.

In the first US pilot study, 15 patients with moderate to severe (CDAI 220-450) active CD received 5 Adacolumn sessions (1/week) [42]. 11 of 15 patients were on steroids ( 9 patients on prednisone and 2 patients on budesonide), 11/15 patients were on azathioprine/6-mercaptopurine, and 10/15 were on infliximab at baseline. No changes in the dosage of these drugs were allowed in the 4-8 weeks before the entry in this study. No severe adverse events were reported during the study. 14 of 15 patients completed the 5-session treatment. 9 of 15 (60\%) 
Table 2. Studies evaluating efficacy of Adacolumn in the treatment of CD patients ('overall response' includes both response and remission rate)

\begin{tabular}{|c|c|c|c|c|}
\hline Group (first author) & Year & Indication & Patients & $\begin{array}{l}\text { Overall } \\
\text { response, \% }\end{array}$ \\
\hline Matsui [47] & 2003 & Active $\mathrm{CD}$, refractory to conventional treatments & 7 & $71(5 / 7)$ \\
\hline Kusaka [48] & 2004 & Active $\mathrm{CD}$, refractory to conventional treatments & 6 & $67(4 / 6)$ \\
\hline Fukuda [49] & 2004 & Active $\mathrm{CD}$, refractory to conventional treatments & 21 & $52(11 / 21)$ \\
\hline Domenech [36] & 2004 & Steroid-dependent CD & $12\left(10^{1}\right)$ & $70(7 / 10)$ \\
\hline Bresci [41] & 2006 & Steroid-resistant CD & 6 & $83(5 / 6)$ \\
\hline Sands [42] & 2006 & Active CD, refractory to conventional treatments & 15 & $60(9 / 15)$ \\
\hline Muratov [50] & 2006 & Active $\mathrm{CD}$, refractory to conventional treatments & 7 & $57(4 / 7)$ \\
\hline Petermann [51], abstract & 2005 & Active CD, steroid-dependent, AZA-resistant & 18 & $44(8 / 18)$ \\
\hline Marin [52], abstract & 2005 & Active $\mathrm{CD}$, refractory or intolerant to conventional drugs & $10\left(9^{1}\right)$ & $66(6 / 9)$ \\
\hline
\end{tabular}

${ }^{1}$ Number of patients included in the final analysis.

patients responded to the treatment (decrease of CDAI of at least 70 points with respect to baseline) and 6/15 (40\%) patients achieved clinical remission $(\mathrm{CDAI}<150)$. The mean CDAI fell from 308.0 points at baseline to 200.6 at week 7.

Among 7 patients with active $\mathrm{CD}$, refractory to conventional treatments, including steroids and infliximab, median CDAI fell from 290 at baseline to 129 at 12 months $(\mathrm{p}=0.016)$, after receiving Adacolumn sessions (1/week) for 5 consecutive weeks [50]. 4 of 7 patients also achieved clinical remission (CDAI <150) at 12 months of follow-up.

A number of studies evaluating efficacy of Adacolumn in the treatment also of $\mathrm{CD}$ patients have been presented at the Digestive Disease Week and the United European Gastroenterology Week in the last 2 years.

In a German study, 18 patients with active (CDAI 200400) steroid-dependent (at least prednisolone $10 \mathrm{mg} /$ day for 6 months) CD received Adacolumn sessions twice a week for the first 3 weeks and then weekly for a further 3 weeks [51]. All patients had failed to respond to azathioprine and 4/18 also did not respond to infliximab. At the end of treatment, 8/18 patients achieved clinical remission (CDAI <150) together with endoscopic improvement and complete withdrawal of steroids.

Lastly, 10 patients with active CD (CDAI $262.1 \pm 64.2)$ were enrolled in an open study because of intolerance to azathioprine/6-mercaptopurine $(\mathrm{n}=3)$, incomplete or no response to a full dosage of steroids and immunosuppressive drugs $(n=5)$ and presence of perianal or enterocutaneous fistulas with no or partial response to immunosuppressive drugs and infliximab $(\mathrm{n}=2)$ [52]. 5 patients en- tered the study due to steroid-dependency, with a mean prednisone dose of $27.6 \pm 19.7 \mathrm{mg} /$ day. All patients received a full course of apheresis treatment (1 session/week for 5 consecutive weeks) and were followed up for at least 6 months. During follow-up, introduction of new drugs or increase in the dosage of concomitant drugs were not allowed. 9 of 10 patients were included in the final analysis. At week 6, 6/9 (66\%) patients achieved clinical remission (CDAI <150), with the CDAI score decreasing from $262 \pm 64.2$ at baseline to $201 \pm 61.3$ after treatment. Among the 5 steroid-dependent patients, the mean prednisone dose decreased from $27.6 \pm 19.7$ to $18.6 \pm 12.8$ $\mathrm{mg} /$ day at week 6 . Only $2 / 6$ patients maintained their clinical remission at 6 months of follow-up. No changes in fistulas activity were observed in patients with penetrating or perianal CD.

\section{Pediatric Inflammatory Bowel Disease}

As far as pediatric IBD patients are concerned, in a first experience, 12 steroid-refractory active UC patients (aged $12.2 \pm 3.1$ years) received 1 Adacolumn session/ week for 5-10 consecutive weeks [53]. Among the 12 patients, 2 had a mild attack of colitis, 2 a moderate attack, 7 had severe colitis and 1 a fulminant colitis. At $24.3 \pm$ 11.5 days after the start of GMA treatment, 8/12 patients achieved clinical remission. The dose of prednisolone per patient decreased from $25.8 \pm 4.9 /$ day at entry to $13.8 \pm$ $3.2 \mathrm{mg}$ at week $5(\mathrm{p}<0.01)$. 4 of $8(50 \%)$ responders relapsed $3.5 \pm 2.2$ months after the last apheresis session. No severe adverse events occurred during the study. 
More recently, 4 pediatric UC patients ( 3 males and 1 female; aged 11-17 years) received 1 Adacolumn session/ week for 5 consecutive weeks [54]. 3 patients had pancolitis and 1 had ulcerative proctitis. 2 patients were steroiddependent and 2 were steroid-refractory. 2 of $4(50 \%)$ patients showed an excellent response after 2 courses of Adacolumn; 1 patient showed a good response to 1 course of Adacolumn, but bloody stools persisted; in 1 patient Adacolumn was ineffective and total colectomy was performed [54].

\section{The Cellsorba Leukocyte Removal Filter}

The Cellsorba leukocyte removal filter is made of polyester fibers that are able to remove a large amount of white blood cells from peripheral blood [6, 7]. Leukocyte apheresis with Cellsorba needs two venous accesses with 18-gauge needles, e.g. at the antecubital (or femoral) vein on one hand for blood supply into the column and on the other arm for returning the blood back to the patient. To prevent coagulation, the blood pumped out from patients' veins is treated with continuous injections of an anticoagulant (heparin or nafamostat mesilate). The anticoagulated blood is passed through a pre-filter made of $10-40 \mu \mathrm{m}$ of fabric and then through the main filter made of finer fiber. Apheresis with Cellsorba is mainly performed once weekly for 5 consecutive weeks. The duration of each session is about $60 \mathrm{~min}$, with 2-3 liters of blood processed at a flow rate of $30-50 \mathrm{ml} / \mathrm{min}$ [6].

Cellsorba is able to remove about $90-100 \%$ of granulocytes and monocytes, 30-60\% of lymphocytes and a certain amount of platelets ( $30 \%$ in the first 30 min of session) from peripheral blood $[6,7]$. Cell removal begins when blood comes into contact with polyester filter. The overall number of leukocytes in peripheral blood increases to $170 \%$ of the initial value $20 \mathrm{~min}$ after the apheresis ('overshoot phenomenon'), but returns to baseline value the next day. This overshoot phenomenon is probably due to a large amount of immature leukocytes entering the peripheral blood from the bone marrow pool [55-57].

As for Adacolumn, for Cellsorba the selective removal of leukocytes from peripheral blood is also probably not the exclusive mechanism responsible for the clinical efficacy of apheresis. In IBD patients responding to Cellsorba apheresis, pro-inflammatory cytokines, such as TNF- $\alpha$, IL-2, IL- 8 and IFN- $\gamma$, showed baseline values near to the normal upper limits. These values normalized after apheresis treatment, such as C-reactive protein and erythrocyte sedimentation rate [55-58]. Flow cytometry also revealed the ability of the Cellsorba system to remove activated cells and adhesion molecule-positive cells [57]. Filter-passed lymphocytes also showed a greater production of IL-4 with respect to pre-apheresis values [59].

As far as the number of CD4+ and CD8+ T cells and the number of $\mathrm{CD} 45 \mathrm{RO}+\mathrm{CD} 4+$ memory $\mathrm{T}$ cells are concerned, the average number of lymphocytes, both $\mathrm{T}$ and B cells, significantly decreases after Cellsorba [60]. The CD4/CD8 ratio does not change after Cellsorba, while the Th1/Th2 ratio seems to significantly decrease [60]. Leukocytapheresis with leukocyte removal filter also seems to be able to selectively remove from peripheral blood the CD14(dull)CD16+ monocytes which represent an important source of TNF $\alpha$ and IL-12. From this point of view, Cellsorba may be presented as an extracorporeal anti-TNF $\alpha$ therapy [61].

All these mechanisms seem to contribute to the clinical efficacy of Cellsorba in the treatment of IBD. The transient lymphopenia observed during Cellsorba leukocytapheresis treatment could potentially represent a limit for this medical device, due to the evidence that lymphopenia could promote autoimmunity development $[62,63]$.

\section{Cellsorba Leukocytapheresis for Ulcerative Colitis}

At the present time a series of open-label studies, mostly involving Japanese centers and only one doubleblind RCT, have evaluated the efficacy and safety of Cellsorba apheresis in UC patients (table 3). No severe adverse events were reported in the following studies.

In a first open clinical trial involving Japanese centers, 13 IBD patients with active disease ( 8 with UC and 5 with CD) were selected for Cellsorba leukocytapheresis treatment [55]. Each patient received an intensive treatment with 1 apheresis session/week for 5 consecutive weeks, followed by a maintenance treatment consisting of 1 session/month for 5 months. During the 5 -week intensive treatment, a clinical response was observed in 11 of 13 (84.6\%) IBD patients, with 6/8 (75\%) UC patients and 5/5 (100\%) CD patients. 8 of 13 patients (61.5\%) maintained clinical response during the 5-month maintenance treatment, without requiring additional therapies.

In a follow-up open study carried out by the same authors, 45 UC patients received an intensive treatment with 1 apheresis session/week for 5 consecutive weeks, followed by a maintenance treatment [57]. 21 patients had a severe attack of UC, 15 patients a moderately severe attack and 9 patients a mild attack of the disease. During 
intensive treatment, 35/45 (77.8\%) patients showed clinical improvement and were allowed to taper concomitant steroid therapy. Improvement was maintained by $32 / 45$ (71.1\%) patients during a 5-month maintenance treatment.

In a multicenter non-blinded RCT, 76 patients with active UC were randomized to receive weekly leukocytapheresis $(n=39)$, added to concomitant therapies, or a high dose of prednisolone $(n=37)$ [64]. The patients in the first group received leukocytapheresis 1 session/week for 5 consecutive weeks and then 1 session/month as maintenance therapy; in patients on steroids the baseline dosage of drugs was maintained but not increased. The patients in the second group with moderately severe UC or severe UC received prednisolone $30-40$ or $60-80 \mathrm{mg} /$ day, respectively. The tapering of steroids started after the first 2 weeks of treatment. No dosage $>15 \mathrm{mg} /$ day of prednisolone was allowed during maintenance therapy. At the end of the study a greater proportion of patients in the Cellsorba group experienced a clinical response with respect to high-dose prednisolone group (74 vs. $38 \%$; $\mathrm{p}=$ 0.005). At the same time the Cellsorba group showed a lower incidence of adverse events ( 24 vs. $68 \%$; $p<0.001$ ) [64]. More interestingly, 6 patients with severe or fulminant UC (average CAI 19.5 points) with toxic megacolon received Cellsorba apheresis 3 times/week for 2 consecutive weeks, followed by 4 further weekly sessions in the next 4 weeks [64]. Of 6 patients, 4 achieved clinical remission, with their average CAI decreasing from 19.5 to 1 point. The remaining 2 patients did not respond to Cellsorba apheresis and underwent colectomy [65].

In the first and at present only multicenter doubleblind RCT with sham column as placebo treatment, 25 UC patients with moderately severe or severe disease not responding to intravenous steroids were randomized to receive leukocytapheresis with Cellsorba or sham device [66]. Nafamostat mesilate was used as anticoagulant both in the active group and placebo group. Each patient received 1 apheresis session/week for 5 consecutive weeks (or 2 sessions/week in the first week if required by the severity of the disease), followed by two further sessions in the next 4 weeks. The patients in both groups were allowed to receive prednisolone orally, at a baseline dose of $15-20$ or $30-40 \mathrm{mg} /$ day for moderately severe and severe UC, respectively. Only after week 2 of treatment was tapering of steroids allowed.

Only 19 patients were included in the final analysis (10 in the active group and 9 in the sham group). In the active group, 10/10 (100\%) patients had moderately severe UC compared to $7 / 9(77.7 \%)$ patients in the sham group. Mean
CAI at baseline was $11.4 \pm 3.2$ in the active group and $10.2 \pm 2.6$ in the sham group. Considering both 'excellent' and 'moderate' improvement, 8/10 (80\%) patients in the active group responded to the treatment compared to $3 / 9(33.3 \%)$ patients in the sham group $(\mathrm{p}<0.05)$. Mean CAI decreased from $11.4 \pm 3.2$ to $4.0 \pm 2.9(\mathrm{p}=0.002)$ in the active group and from $10.2 \pm 2.6$ to $7.2 \pm 5.2(\mathrm{p}=$ $0.24)$ in the sham group. However, the difference in improvement of CAI among the two groups did not reach statistical significance $(\mathrm{p}=0.054)$. As far as the endoscopic response is concerned, a complete mucosal healing was observed in 60 and $22 \%$ of patients in the active and in the sham group, respectively. In the active group the mean dose of prednisolone was reduced from $22.0 \pm 3.9$ to $7.8 \pm 9.2 \mathrm{mg} / \mathrm{day}$ at week 10 . On the other hand, in the sham group the mean dose of prednisolone decreased from $27.9 \pm 8.3$ to $19.4 \pm 12.8 \mathrm{mg} /$ day. The difference between the two groups was statistically significant $(\mathrm{p}<$ 0.01 ). A total of 4 patients in the sham group experienced adverse events compared to only 1 patient in the active group [66].

Very recently in a randomized pilot trial, 20 patients with active UC not responding to conventional treatment received Cellsorba apheresis treatment, 1 session/week for 5 consecutive weeks [67]. Of 20 patients, 14 achieved clinical remission and $6 / 20$ patients also achieved endoscopic remission. The 14 responder patients were further randomized to receive a monthly apheresis session or no further treatment. After 6 months, 5/8 patients receiving monthly apheresis maintained remission compared to $1 / 6$ in the group with no further treatment [67].

\section{Cellsorba Leukocytapheresis for Crohn's Disease}

A very small amount of data are available on the efficacy of Cellsorba in the treatment of CD. Results of studies evaluating the efficacy of Cellsorba in the treatment of both UC and CD are summarized in table 3. We have previously reported the preliminary data by Sawada et al. [55]. In another small study, 18 patients with active CD received leukocytapheresis induction treatment (1 session/week for 5 consecutive weeks), followed by a maintenance treatment (1 session/month) [68]. At the end of induction treatment, 9/18 (50\%) patients achieved clinical remission. Only patients achieving remission also showed a significant decrease in C-reactive protein and erythrocyte sedimentation rate values with respect to baseline. 
Table 3. Studies evaluating efficacy of Cellsorba in the treatment of IBD patients ('overall response' includes both response and remission rate)

\begin{tabular}{lllcc}
\hline Group (first author) & Year & Indication & Patients & Overall response, \% \\
\hline Sawada [55] & 1995 & Active IBD & 8 UC & $75(6 / 8)$ \\
& & & 5 CD & $100(5 / 5)$ \\
Sawada [57] & 1997 & Active mild to severe UC & 45 & $77.8(35 / 45)$ \\
Sawada [64] & 2003 & Active UC & 39 & $74(29 / 39)$ \\
Sawada [65] & 2005 & Severe/fulminant UC with toxic megacolon & $66.6(4 / 6)$ \\
Sawada [66] & 2005 & Moderate-to-severe UC, refractory to i.v. steroids & 10 active apheresis & $80(8 / 10)$ \\
Emmrich [67] & 2007 & UC refractory to conventional drugs & 9 sham apheresis & $33.3(3 / 9)$ \\
Kosaka [68] & 1999 & CD refractory to conventional drugs & 20 & $70(14 / 20)$ \\
\hline
\end{tabular}

${ }^{1}$ Double-blind RCT.

\section{Conclusions}

At the present time, management of moderate and severe forms of IBD is far from being fully satisfactory, especially for the many cases of steroid dependency, steroid resistancy and steroid intolerance. A large number of biological therapies targeting cytokines involved in intestinal inflammation have been approved for the treatment of moderate and severe forms of both CD and UC, with not fully satisfactory results in terms of efficacy and costs. Furthermore, new concerns about the safety of biological therapies are emerging, especially regarding long-term safety and the risk of developing neoplasia or severe infections.

Cytapheresis is a safe treatment for the management of IBD. Currently a too little number of Oxford Evidence Level IIb RCTs as well as abundant level III and IV evi- dence suggest the efficient use of cytapheresis for UC, both in steroid-dependent/-refractory and steroid-naive patients. To date, a comparably limited number of studies on $\mathrm{CD}$ with small numbers of patients do not yet yield the same strength of evidence for the use of cytapheresis in $\mathrm{CD}$. Considering the extreme protocol heterogeneity of the studies, the limited number of patients and the lack of RCT evaluating use of Adacolumn for UC, it remains difficult to establish which role apheresis may play in the management of IBD [69]. Future larger randomized controlled trials, possibly involving different ethnic groups, should further definitively establish the efficacy of cytapheresis in IBD, increasing the available levels of evidence and helping to establish its exact indications and limits.

\section{References}

1 Present DH: How to do without steroids in inflammatory bowel disease. Inflamm Bowel Dis 2000;6:48:57.

$>2$ Nikolaus S, Bauditz J, Gionchetti P: Increased secretion of pro-inflammatory cytokines by circulating polymorphonuclear neutrophils and regulation by interleukin-10 during intestinal inflammation. Gut 1998; 42:470-476.

3 Fiocchi C: Inflammatory bowel disease: etiology and pathogenesis. Gastroenterology 1998;115:182-205.
4 Bicks RO, Groshart KD: Editorial: the current status of T-lymphocyte apheresis treatment of Crohn's disease. J Clin Gastroenterol 1989;11:136-138.

5 Saniabadi AR, Hanai H, Takeuchi K, et al: Adacolumn, an adsorptive carrier based granulocyte and monocyte apheresis device for the treatment of inflammatory and refractory diseases associated with leukocytes. Ther Apher Dial 2003;7:48-59.

6 Shirokaze J: Leukocytapheresis using a leukocyte removal filter. Ther Apher 2002;6: 261-266.
7 Shibata H, Kuriyama T, Yamawaki N: Cellsorba. Ther Apher Dial 2003;7:44-47.

-8 Ashizuka S, Nishiura R, Ishikawa N, et al: Leukocytapheresis for ulcerative colitis: a comparative study of anticoagulant (nafamostat mesilate vs. dalteparin sodium) for reducing clinical complications. Ther Apher Dial 2006; 10:54-58.

$\checkmark 9$ Hiraishi K, Takeda Y, Saniabadi A, et al: Studies on the mechanisms of leukocytes adhesion to cellulose acetate beads: an in vitro model to assess the efficacy of cellulose acetate carrier-based granulocyte and monocyte adsorptive apheresis. Ther Apher Dial 2003;7:334-340. 
10 D’Arrigo C, Candal-Couto JJ, Greer M, et al: Human neutrophil $\mathrm{Fc}$ receptor mediated adhesion under flow: a hallow fiber model of intravascular arrest. Clin Exp Immunol 1994;100:173-179.

-11 Kashiwagi N, Saniabadi A, Shimoyama T: Immunomodulatory effects of granulocyte and monocyte adsorption apheresis as a treatment for patients with ulcerative colitis. Dig Dis Sci 2002;47:1334-1341.

12 Shimoyama T, Sawada K, Saniabadi A: Safety and efficacy of granulocyte and monocyte apheresis in patients with active ulcerative colitis. J Clin Apher 2001;16:1-9.

13 Hanai H, Watanabe F, Saniabadi A, et al: Therapeutic efficacy of granulocyte and monocyte adsorption apheresis in severe active ulcerative colitis. Dig Dis Sci 2002;47: 2349-2353.

14 Andoh A, Tsujikawa T, Inatomi O, et al: Suppression of inflammatory cytokine secretion by granulocyte/monocyte adsorptive apheresis in active ulcerative colitis. Ther Apher Dial 2005;9:123-127.

15 Rembacken BJ, Newbould HE, Richards SJ, et al: Granulocyte apheresis in inflammatory bowel disease: possible mechanism of effect. Ther Apher 1998;2:93-96.

16 Kashiwagi N, Hirata I, Kasukawa R: Granulocyte/active oxygen and modulation of the inflammation response. J Act Oxyg Free Rad 1995;6:1-25.

17 Jutila MA, Rott I, Berg EL, et al: Function and regulation of the neutrophil MEL-14 antigen in vivo: comparison LFA-1 and Mac-1. J Immunol 1989;143:3318-3324.

18 Dransfield I, Stocks SG, Haslett C: Regulation of cell adhesion molecule expression and function associated with neutrophil apoptosis. Blood 1995;85:3246-3253.

19 Takeda Y, Hiraishi K, Takeda H, et al: Cellulose acetate beads induce release of interleukin-1 receptor antagonist, but not tumor necrosis factor- $\alpha$ or interleukin- $1 \beta$ in human peripheral blood. Inflam Res 2003;52: 287-290.

20 Sakimura K, Omori T, Iwashita E, et al: Clinical response is associated with elevated plasma interleukin-1 receptor antagonist during selective granulocyte and monocyte apheresis in patients with ulcerative colitis. Dig Dis Sci 2006;51:1525-1531.

21 Saniabadi AR, Hanai H, Suzuki Y, et al: Adacolumn for selective leukocytapheresis as a non-pharmacological treatment for patients with disorders of the immune system: an adjunct or an alternative to drug therapy? J Clin Apher 2005;20:171-184.

22 Hanai H, Watanabe F, Yamada M, et al: Correlation of serum soluble TNF- $\alpha$ receptor I and II levels with disease activity in patients with ulcerative colitis. Am J Gastroenterol 2004;99:1532-1538.
23 Suzuki Y, Aoki H, Nakamura K, et al: Adacolumn selective leukocytapheresis in patients with active ulcerative colitis: clinical efficacy, effects on plasma interleukin-8 and the expression of Toll-like receptors on granulocytes (abstract). Gastroenterology 2006; 130(Suppl 2):A-657.

24 Fukunaga K, Yokoyama Y, Fukuda Y, et al: Demonstration of low regulatory CD25high+CD4+ and high pro-inflammatory CD28-CD4+ T-cells subsets in patients with ulcerative colitis: modified by selective granulocyte and monocyte adsorption apheresis (abstract). Gut 2006;55(Suppl V): A-125.

25 Hanai $\mathrm{H}$, Iida $\mathrm{T}$, Watanabe $\mathrm{F}$, et al: Elevated pro-inflammatory monocytes, $\mathrm{CD} 14+\mathrm{CD} 16+$ in the peripheral blood of patients with active inflammatory bowel disease decreases markedly during Adacolumn leukocytapheresis therapy (abstract). Gut 2006;55(Suppl V):A-104.

26 Shimoyama T, Sawada K, Tanaka T, et al: Granulocyte and monocyte apheresis with the G-1 column in the treatment of patients with active ulcerative colitis (in Japanese). Jpn J Apher 1999;18:117-131.

27 Suzuki Y, Yoshimura N, Saitoh Y, et al: Gran ulocyte and monocyte adsorptive apheresis in a case with severe ulcerative colitis unresponsive to conventional medication. J Clin Apher 2003; 18:40-42.

28 Nakamura T, Suzuki Y, Koide H, et al: Granulocyte and monocyte adsorption apheresis in a patient with antiglomerular basement membrane glomerulonephritis and active ulcerative colitis. Am J Med Sci 2003;325: 296-298.

29 Fukunaga K, Sawada K, Fukuda Y, et al: Extracorporeal monocyte granulocytapheresis was effective for a patient of erythema nodosum concomitant with ulcerative colitis. Ther Apher Dial 2003;7:122-126.

30 Premchand P, Takeuchi K, Byarnason I: Granulocyte, macrophage, monocyte apheresis for refractory ulcerative proctitis. Eur J Gastroenterol Hepatol 2004;16:943-945.

31 Hanai H, Watanabe F, Takeuchi K, et al: Leukocyte adsorptive apheresis for the treatment of active ulcerative colitis: a prospective, uncontrolled, pilot study. Clin Gastroenterol Hepatol 2003;1:28-35.

32 Suzuki Y, Yoshimura N, Saniabadi AR, et al: Selective granulocyte and monocyte adsorptive apheresis as a first-line treatment for steroid naive patients with active ulcerative colitis: a prospective uncontrolled study. Dig Dis Sci 2004;49:565-571.

33 Naganuma M, Funakoshi F, Sakuraba A, et al: Granulocytapheresis is useful as an alternative therapy in patients with steroid-refractory or -dependent ulcerative colitis. Inflamm Bowel Dis 2004;10:251-257.
34 Hanai H, Watanabe F, Yamada M, et al: Adsorptive granulocyte and monocyte apheresis versus prednisolone in patients with corticosteroid-dependent moderately severe ulcerative colitis. Digestion 2004;70:36-44.

-35 Yamamoto T, Umegae S, Kitagawa T, et al: Granulocyte and monocyte adsorptive apheresis in the treatment of active distal ulcerative colitis: a prospective, pilot study. Aliment Pharmacol Ther 2004;20:783-792.

$\checkmark 36$ Domenech E, Hinojosa H, Esteve-Comes M, et al: Granulocyteapheresis in steroid-dependent inflammatory bowel disease: a prospective, open, pilot study. Aliment Pharmacol Ther 2004;20:1347-1352.

37 Kanke K, Nakano M, Hiraishi H, et al: Clinical evaluation of granulocyte/monocyte apheresis therapy for active ulcerative colitis. Dig Liver Dis 2004;36:811-817.

38 Kim HJ, Kim JS, Han DS, et al: Granulocyte and monocyte adsorption apheresis in Korean conventional treatment-refractory patients with active ulcerative colitis: a prospective open-label multicenter study. Korean J Gastroenterol 2005;45:34-44.

39 Kruis W, Dignass A, Steinhagen-Thiessen E, et al: Open label trial of granulocyte apheresis suggests therapeutic efficacy in chronically active steroid-refractory ulcerative colitis. World J Gastroenterol 2005;11:70017006 .

40 D'Ovidio V, Aratari A, Viscido A, et al: $\mathrm{Mu}$ cosal features and granulocyte-monocyte apheresis in steroid-dependent/refractory ulcerative colitis. Dig Liver Dis 2006;38:389394.

41 Bresci G, Parisi G, Bertoni M, et al: Treatment of active steroid-refractory inflammatory bowel diseases with granulocytapheresis: our experience with a prospective study. World J Gastroenterol 2006;12:2201-2204.

42 Sands BE, Sandborn WJ, Wolf DC, et al: Pilot feasibility studies leukocytapheresis with the Adacolumn apheresis system in patients with active ulcerative colitis and Crohn's disease. J Clin Gastroenterol 2006;40:482-489.

43 Sakuraba A, Inoue N, Kohgo Y, et al: A multicenter, randomized, controlled trial between weekly and semiweekly treatment with granulocyte and monocyte adsorption apheresis for active ulcerative colitis (abstract). Gastroenterology 2005;128(Suppl 2): A-585.

44 Tanaka T, Okanobu H, Saniabadi AR, et al: Efficacy of Adacolumn-selective leukocytapheresis in patients with steroid-naive and steroid-dependent with moderate to severe ulcerative colitis (abstract). Gastroenterology 2006;130(Suppl 2):A-126.

45 Hanai $\mathrm{H}$, Iida $\mathrm{T}$, Watanabe $\mathrm{F}$, et al: Intensive granulocyte and monocyte apheresis versus intravenous prednisolone in patients with severe ulcerative colitis: a multicentre randomized controlled study (abstract). Gut 2006;55(Suppl V):A-40. 
46 Sakuraba A, et al: An open-labeled trial of granulocyte and monocyte adsorption apheresis for pouchitis (abstract). Gastroenterology 2006;130(Suppl 2):A-661.

$\checkmark 47$ Matsui T, Nishimura T, Matake H, et al: Granulocytapheresis for Crohn's disease: a report on seven refractory patients. Am J Gastroenterol 2003;98:511-512.

-48 Kusaka T, Fukunaga K, Ohnishi K, et al: Adsorptive monocyte-granulocytapheresis (M-GCAP) for refractory Crohn's disease. J Clin Apher 2004;19:168-173.

49 Fukuda Y, Matsui T, Suzuki Y, et al: Adsorptive granulocyte and monocyte apheresis for refractory Crohn's disease: an open multicenter prospective study. J Gastroenterol 2004;39:1158-1164.

50 Muratov V, Lundahl J, Ulfgren AK, et al: Down-regulation of interferon- $\gamma$ parallels clinical response to selective leukocyte apheresis in patients with inflammatory bowel disease: a 12-month follow-up study. Int J colorectal Dis 2006;21:493-504.

51 Petermann S, Rieckeheer K, Kury C, et al: Leukocytapheresis using granulocyte and monocyte adsorption apheresis in chronic active Crohn's disease. Gut 2005;54:A-177.

52 Marin I, Taxonera C, Abreu L, et al: Granulocyteapheresis in difficult-to-treat Crohn's disease: a multicenter prospective open study. Gut 2005;54:A158.

53 Tomomasa T, Kobayashi A, Kaneko H, et al: Granulocyte adsorptive apheresis for pediatric patients with ulcerative colitis. Dig Dis Sci 2003;48:750-754
54 Ikeda H, Ishimaru H, Takayasu H, et al: Efficacy of granulocyte apheresis in pediatric patients with ulcerative colitis: a pilot study. J Pediatr Gastroenterol Nutr 2006;43:592596.

55 Sawada K, Ohnishi K, Fukui S, et al: Leukocytapheresis therapy, performed with leukocyte removal filter for inflammatory bowel disease. J Gastroenterol 1995;30:322-329.

56 Sawada K, Ohnishi K, Kosaka T, et al: Leukocytapheresis therapy with leukocyte removal filter for inflammatory bowel disease. J Gastroenterol 1995;30(suppl 8):124-127.

57 Sawada K, Ohnishi K, Kosaka, et al: Leukocytapheresis with leukocyte removal filter as a new therapy for ulcerative colitis. Ther Apher 1997;1:207-211.

58 Andoh A, Ogawa A, Kitamura K, et al: Suppression of interleukin-1 $\beta$ and tumor necrosis factor- $\alpha$ induced inflammatory responses by leukocytapheresis therapy in patients with ulcerative colitis. J Gastroenterol 2004; 39:1150-1157.

59 Noguchi M, Hiwatashi N, Hayakawa T, et al Leukocyte removal filter-passed lymphocytes produces large amounts of interleukin4 in immunotherapy for inflammatory bowel disease: role of bystander suppression. Ther Apher 1998;2:109-114.

60 Andoh A, Tsujikawa T, Inatomi O, et al: Leukocytapheresis therapy modulates circulating T-cell subsets in patients with ulcerative colitis. Ther Apher Dial 2005;9:270-279.

61 Kanai T, Makita S, Kavamura T, et al: Extracorporeal elimination of $\mathrm{TNF} \alpha$-producing CD14(dull)CD16 ${ }^{+}$monocytes in leukocytapheresis therapy for ulcerative colitis. Inflamm Bowel Dis 2007;13:284-290.
62 King C, Ilic A, Koelsch K, et al: Homeostatic expansion of $\mathrm{T}$ cells during immune deficiency. Cell 2004;117:265-277.

63 Kanai T, Hibi T, Watanabe M: The logics of leukocytapheresis as a natural biological therapy for inflammatory bowel disease. Expert Opin Biol Ther 2006;6:453-466.

64 Sawada K, Muto T, Shimoyama T, et al: Multicenter randomized controlled trial for the treatment of ulcerative colitis with a leukocytapheresis column. Curr Pharm Des 2003; 9:307-321.

-65 Sawada K, Egashira A, Ohnishi K, et al: Leukocytapheresis for management of fulminant ulcerative colitis with toxic megacolon. Dig Dis Sci 2005;50:767-773.

-66 Sawada K, Kusugami K, Suzuki Y, et al: Leukocytapheresis in ulcerative colitis: results of a multicenter double-blind prospective casecontrol study with sham apheresis as placebo treatment. Am J Gastroenterol 2005;100: 1362-1369.

-67 Emmrich J, Petermann S, Nowak D, et al: Leukocytapheresis in the management of chronic active ulcerative colitis. Results of a randomized pilot trial. Dig Dis Sci 2007;52: 2044-2053.

68 Kosaka T, Sawada K, Ohnishi K, et al: Effect of leukocytapheresis therapy using leukocyte removal filter in Crohn's disease. Intern Med 1999;38:102-111.

69 Hibi T, Sakuraba A: Is there a role for apheresis in gastrointestinal disorders? Nat Clin Pract Gastroenterol Hepatol 2005;2:200201. 